\title{
First Cycle Neutrophil Count and Subsequent Chemotherapy Dose Delivery in Breast Cancer Patients Receiving Adjuvant Chemotherapy
}

\author{
Ahmed Ashour*, Gamal El-Husseiny, Amr Abdel Aziz, Waleed O. Arafat \\ Department of Clinical Oncology and Nuclear Medicine, Faculty of Medicine, Alexandria University, Alexandria, Egypt. \\ Email: ${ }^{*}$ asure_egypt@yahoo.com \\ Received December $5^{\text {th }}, 2013$; revised January $5^{\text {th }}, 2014$; accepted January $13^{\text {th }}, 2014$ \\ Copyright (C) 2014 Ahmed Ashour et al. This is an open access article distributed under the Creative Commons Attribution License, \\ which permits unrestricted use, distribution, and reproduction in any medium, provided the original work is properly cited. In accor- \\ dance of the Creative Commons Attribution License all Copyrights (C) 2014 are reserved for SCIRP and the owner of the intellectual \\ property Ahmed Ashour et al. All Copyright (C) 2014 are guarded by law and by SCIRP as a guardian.
}

\section{ABSTRACT}

Background: Chemotherapy-induced neutropenia is a major dose-limiting toxicity of systemic cancer chemotherapy, and it is associated with substantial morbidity, mortality, and costs. The ability to predict the group of patients who are at high risk for neutropenic complications might help to reduce the morbidity due to febrile neutropenia and increase the likelihood of delivering full chemotherapy dose on time. In addition, hematopoietic growth factors could be administered to patients who need them and allow more efficient use of medical resources. Methods: The study included 120 female patients with breast cancer, treated in adjuvant setting with FAC chemotherapy regimen. Chemotherapy was delayed if the ANC was less than 1500 cells/ $\mu \mathrm{L}$ on the planned first day of the cycle. Dose delays would be for 1 week. The chemotherapy dose was reduced if the patient experienced an episode of febrile neutropenia. Dose reductions usually consist of a $20 \%$ reduction in dosage of all chemotherapy drugs. Results: Out of the 120 patients in the study, 58 patients experienced neutropenic complications (febrile neutropenia or dose delayed) accounting for $\mathbf{4 8 . 3 \%}$ of total sample size. In univariate analysis, there was statistically significant association between first cycle ANC nadir and neutropenic complication $(p=$ 0.0001). There was also statistically significant association between pretreatment first cycle ANC (p $=0.001)$, pretreatment platelets count $(p=0.054)$, first cycle platelets count nadir $(p=0.041)$, number of involved lymph nodes $(p=0.031)$ and the occurrence of varies neutropenic complications. However, in multivariate analysis, ANC nadir was only the independent risk factor for occurrence of neutropenic. Conclusions: The first cycle absolute neutrophil counts nadir provides useful information on the risk of future neutropenia, febrile neutropenia and dose delay or reduction with estimated sensitivity and specificity, which were $64.5 \%$ and $77.6 \%$, respectively. Other factors such as pretreatment absolute neutrophil count, number of involved LN, pretreatment platelet count and first cycle platelet nadir can be used to predict occurrence of neutropenic complications in breast cancer patients undergoing chemotherapy. Further studies are needed among different populations with different tumor types and different treatment regimens to confirm the results and explore other factors which may contribute to occurrence of neutropenic complications.

\section{KEYWORDS}

Breast Cancer; Chemotherapy; Neutropenia

\section{Introduction}

Breast cancer is the most common malignancy among women worldwide. It accounts for $32 \%$ of all female cancers:[1] in Egypt. Data reported from Cairo National

"Corresponding author.
Cancer Institute (NCI) showed that between January 2002 and December 2003, there were 3519 new cases of breast cancer which accounted for $19 \%$ of all newly diagnosed proven malignant cases [2].

Breast cancer patients usually treated in multimodality 
approach which includes surgery, radiotherapy, chemotherapy, hormonal treatment and targeted therapy. Adjuvant chemotherapy represents a significant advance in the management of breast cancer. It leads to prolongation of both disease-free and overall survival. Its intent is to eliminate or delay the subsequent appearance of clinically occult micrometastatic diseases which are thought to account for distant treatment failures after local treatment alone [3].

Most chemotherapy regimens used in adjuvant treatment of breast cancer lead to varieties of temporary and reversible side effects. The side effects include myelosuppression (includes neutropenia), mucositis, alopecia, nausea, vomiting, diarrhea, anorexia and localized skin reactions. The chemotherapy toxicities must be considered when assessing the relative risk benefit ratio of adjuvant treatment. In general, the benefit gained in terms of survival exceeds the negative impact of toxicities on patient's quality of life $[4,5]$.

Chemotherapy-induced neutropenia is the major doselimiting toxicity of systemic cancer chemotherapy, and it is associated with substantial morbidity, mortality, and costs. Neutropenia may result in febrile neutropenia, chemotherapy dose delay or reduction of the dose of chemotherapy [6].

Febrile neutropenia is one of a medical emergency. It is responsible for almost $5 \%$ overall mortality rates in patients with solid tumors and $11 \%$ overall mortality rates in patients with hematological malignancies. It is often necessitating hospitalization for evaluation and empiric broad-spectrum antibiotics [7].

Chemotherapy dose delay or reduction in response to severe neutropenia at the time of scheduled chemotherapy leads to reduced dose intensity and potentially compromises treatment outcome and overall survival. This was demonstrated in Bonadonna's study in patients with breast cancer that receives 12 cycles of adjuvant CMF chemotherapy. Adjuvant chemotherapy was compared with surgery alone, and dose reduction was used routinely in the case of toxicity or for older patients. Over a 20 -year follow-up, patients receiving less than $85 \%$ of the intended dose had a markedly poorer survival than those receiving more than $85 \%$ of the intended dose $[8,9]$.

Colony stimulating factors which stimulate the production of neutrophils are used to prevent or reduce the severity of Chemotherapy-induced neutropenia. The American Society of Clinical Oncology (ASCO) guidelines in 2006 recommend the only uses of CSFs for primary prophylaxis when the risk of $\mathrm{FN}$ is expected to exceed $20 \%$ and for secondary prophylaxis to protect and prevent a new episode of febrile neutropenia or dose reduction where patients have experienced these complications with their first cycle of treatment $[10,11]$.

A number of studies have attempted to identify risk factors for neutropenia and its consequences to develop predictive models that are capable of identifying patients at greater risk for such complications. They aim to reduce the morbidity due to febrile neutropenia and increase the likelihood of delivering full chemotherapy dose on time by application of prophylactic CSF supports. In addition, hematopoietic growth factors would be administered to patients who need them that result in cost savings and allow more efficient use of medical resources. But unfortunately, most of these studies were retrospective and conclusive results were limited because of the different study designs, small patients' number and diverse chemotherapy regimens used in these studies.

Silber et al. first published a retrospective model for predicting the risk of neutropenia from first cycle nadir in 1998. These authors reviewed the cases of breast cancer patients who received adjuvant chemotherapy with either FAC (fluorouracil, doxorubicin, and cyclophosphamide) or standard CMF regimens. The study demonstrated statistically significant correlation between first cycle ANC nadir and occurrence of neutropenic complications ( $\mathrm{p}=$ 0.0001). It also demonstrated statistically significant correlation between first cycle hemoglobin nadir and occurrence of neutropenic complications $(p=0.0001)$ [12].

Rivera et al. conducted prospective trial at MD Anderson cancer center to investigate the predictive role of the first cycle nadir in neutrophil count and occurrence of various neutropenic complications. The study was conducted on 143 patients. All patients were treated with the FAC. They demonstrated statistically significant correlation between first cycle ANC nadir and occurrence of neutropenic complications $(p=0.0001)$ [13].

\section{Methods}

\subsection{Study Design}

The study was conducted in prospective single arm design on 120 consecutive patients at clinical oncology and nuclear medicine department faculty of medicine Alexandria University between the periods of $1^{\text {st }}$ March 2009 to $1^{\text {st }}$ March 2010. It aimed to investigate the predictive role of first cycle nadir in neutrophil count and occurrence of various neutropenic complications which include febrile neutropenia, dose delays and dose reduction at the time of scheduled chemotherapy. The impact of these finding will allow better identification of patients at high risk for development neutropenic complication and this may help to reduce the morbidity due to febrile neutropenia and increase the likelihood of delivering full chemotherapy dose on time. 


\subsection{Patients}

All patients would be female below age of 75 years old with the diagnosis of breast cancer stage I, II, III with performance status between 0 to 1 according to WHO scale, with good renal and liver functions and adequate bone marrow reserve (hemoglobin level $\geq 9 \mathrm{~g} / \mathrm{dL}$, absolute neutrophil count $\geq 1500$ cells $/ \mu \mathrm{L}$, platelet count $\geq$ 100000 cells $/ \mu \mathrm{L}$ ) and scheduled to receive FAC chemotherapy regimen in the adjuvant setting. Patient who was pregnant or lactating or had cardiac disease or pervious history of chemotherapy would exclude from study.

All Patients were evaluated pretreatment by history taking, physical examination, laboratory investigations (include complete blood count, liver functions and kidney functions), radiological investigations (chest X-ray, Ultrasonography or CT abdomen and pelvis, bone scan if indicated and Echocardiogram). The informed consent was taken from the patients before the beginning of the study.

\subsection{Treatment}

Primary surgical treatment consisted of a modified radical mastectomy (MRM) or breast conservative surgery (BCS). If indicated, the patient was treated with postoperative local radiotherapy or hormonal therapy. All patients were treated with the FAC regimen.

The FAC regimen was consisted of $500 \mathrm{mg} / \mathrm{m}^{2}$ fluorouracil given intravenously on days $1,50 \mathrm{mg} / \mathrm{m}^{2}$ doxorubicin given intravenously on day 1 and $500 \mathrm{mg} / \mathrm{m}^{2}$ cyclophosphamide given intravenously on day 1, and. The treatment was given every 3 weeks for a total of six cycles.

\subsection{Complete Blood Count}

CBC with differential counts was done on days 8, and 15 of each cycle. CBC variables would collected and tabulated included the total white blood count (WBCs) per microliter, platelet count per microliter, hemoglobin level in grams per deciliter, and the polymorph nuclear cells or neutrophils or segmented cells either in absolute count per microliter or as percentage from WBCs.

\subsection{Chemotherapy Dose Delay or Reduction}

Chemotherapy was delayed if the ANC was being less than 1500 cells $/ \mu \mathrm{L}$ on the planned first day of the cycle. Dose delays would be for 1 week. Twenty percent reduction for all chemotherapy drugs dose was applied if the patient experienced an episode of febrile neutropenia. Colony stimulating factors were not used as primary or secondary prophylaxis. The risk group consists of all patients who experience at least one febrile neutropenic episode or subjected to at least one dose delay during treatment course of chemotherapy.

\subsection{Statistical Analysis}

Risk factors for chemotherapy induce neutropenia (either febrile neutropenia or dose delayed) were tested in univariate and multivariate analysis using the procedures of the SPSS-17 programme (Statistical package for Social Sciences version 17).

The correlation between a clinical or a biological parameter and the incidence of chemotherapy-induced neutropenia wasperformed using the $\mathrm{X}^{2}$ test or the MannWhitney test. Logistic regression including the studied parameters of the univariateanalysis was performed that was statistically significant with occurrence of chemotherapy induce neutropenia using the logistic program of SPSS 17: a forward regression procedure was used with a $P$ value of 0.05 for entry.

\section{Results}

In the present study, patient's age ranged from $30-67$ with mean age of 49.6. There were 34 patients $<50$ years old ( $29 \%$ of total sample size) and 86 patients $\geq 50$ years old (71\% of total sample size) and most of the patients in the study were premenopausal (55\% of total sample size). Table 1 demonstrated the patient characters in the study. Out of 120 patients in the study, 109 patients underwent modified radical mastectomy $(90.8 \%$ of total patients in the study) and 11 patient underwent breast conservative surgery (9.2 of total patient in the study). Tumor size in the study mostly range between $2-5 \mathrm{~cm}$ (51.7\% of total sample size) followed by tumor size of more than $5 \mathrm{~cm}$ (36.7\% of total sample size). Lymph nodes statuses were mostly positive (72.5\% of total population size) and most of them range between 3 - 9 lymph nodes (30.8\% of total population size). Histopathologically there were 107 patients had infiltrating ductal carcinoma (89.2\% of total sample size), 4 patients had infiltrating lobular carcinoma (3.3\% of total sample size), 7 patients had mixed infiltrating ductal and lobular carcinoma (5.8\% of total sample size) and 2 patients had medullary carcinoma (1.7\% of total sample size). Out of 114 patients who had either infiltrating ductal carcinoma or mixed infiltrating ductal and lobular carcinoma, 100 patients had tumor grade 2 (83.3\% of total sample size), 16 patients had tumor grade 3 (8.3\% of total sample size) and 4 patients had tumor grade 1 (3.3\% of total sample size). Hormonal status among patients in the study was mostly positive $(82.5 \%$ of total patients in the study). Table 2 demonstrated the pathological characteristics of tumor.

Out of the 120 patients in the study, 58 patients experienced neutropenic complications (febrile neutropenia or 
Table 1. The patient characters in the study.

\begin{tabular}{ccc}
\hline & Number & Percent \\
\hline Age & & \\
$<\mathbf{5 0}$ & 34 & 29.0 \\
$\mathbf{5 0 0}$ & 86 & 71.0 \\
Site & & \\
Right & 52 & 43.3 \\
Left & 65 & 54.2 \\
Bilateral & 3 & 2.5 \\
Menopausal status & & \\
Pre menopause & 66 & 55.0 \\
Post menopause & 54 & 45.0 \\
\hline
\end{tabular}

Table 2. The pathological characteristics of tumor.

\begin{tabular}{ccc}
\hline & Number & Percent \\
\hline Pathology & 7 & 5.8 \\
IDC and ILC & 107 & 89.2 \\
IDC NOS & 4 & 3.3 \\
ILC & 2 & 1.7 \\
Medullary carcinoma & 4 & \\
GRADE & 100 & 3.3 \\
1 & 16 & 83.3 \\
2 & & 13.3 \\
3 & 14 & \\
T stage & 62 & 11.7 \\
1 & 44 & 51.7 \\
2 & & 36.7 \\
3 & 33 & \\
N stage & 26 & 27.5 \\
0 & 37 & 21.7 \\
1 & 24 & 30.8 \\
2 & & 20.0 \\
3 & 99 & 17.5 \\
ER/PR & & 82.5 \\
Negative & & \\
Positive & & \\
& &
\end{tabular}

dose delayed) accounting for $48.3 \%$ of total sample size, 2 of them had febrile neutropenia and 56 had dose delayed because ANC was less than 1500 cells $/ \mu \mathrm{L}$ on the planned first day of the cycle and this was demonstrated in Figure 1. Among 58 patients developed neutropenic complications, 20 patients developed first neutropenic event in third chemotherapy cycle (34.5\%), 19 patients developed first neutropenic event in second cycle (32.8\%), 9 patients developed first neutropenic event during forth cycle (15.5\%), 7 patients developed first neutropenic event during fifth cycle (12.1\%) and 3 patients developed neutropenic event during sixth cycle (5.1\%).

The analysis had demonstrated that that there was statistically significant association between first cycle ANC

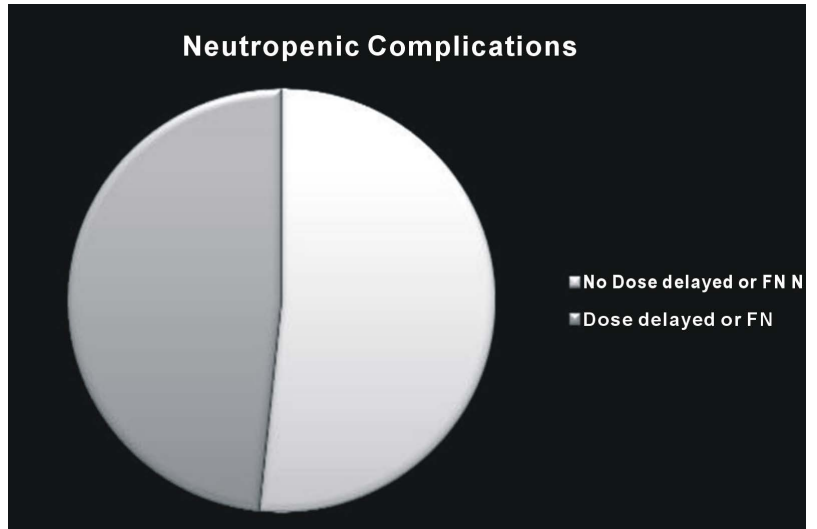

Figure 1. The distribution of neutropenic complications among patients in the study.

nadir and neutropenic complication $(\mathrm{p}=0.0001)$. There was also statistically significant association between pretreatment first cycle ANC $(p=0.001)$, pretreatment platelets count ( $p=0.054)$, first cycle platelets count nadir $(\mathrm{p}=0.041)$, number of involved lymph nodes $(\mathrm{p}=$ 0.031 ) and the occurrence of varies neutropenic complications. ). There was no statistically significant association between tumor size $(\mathrm{p}=0.676)$, patients' age $(\mathrm{p}=$ $0.663)$, menopausal status $(\mathrm{p}=0.342)$, pretreatment hemoglobin level $(p=0.252)$. And first cycle hemoglobin nadir ( $p=0.332$ ) and occurrence of neutropenic complications. This demonstrated in Table 3 . The predictive power of first cycle ANC nadir was demonstrated using ROC curve where area under curve was 0.729 and 95\% CI $(0.637-0.822)$. The sensitivity and specificity obtained at optimal cut off probability of 0.5 were $64.5 \%$ and $77.6 \%$ respectively. This was demonstrated in Figure 2.

Multivariate logistic regression was used to identify independent risk factors using the parameters of the univariate analysis that was statistically significant association with neutropenic complications (pretreatment first cycle ANC, pretreatment first cycle platelets count, first cycle platelets count nadir, first cycle ANC nadir, and lymph nodes) showed that the first cycle ANC nadir was only independent risk factor for occurrence of neutropenic complications. This was demonstrated in Tables 4 and 5.

\section{Discussion}

The ability to predict the group of patients who are at high risk for neutropenic complications might help to reduce the morbidity due to febrile neutropenia and increase the likelihood of delivering full chemotherapy dose on time. In addition, hematopoietic growth factors could be administered to patients who need them and allow more efficient use of medical resources. A number of studies have attempted to identify risk factors for neu- 
Table 3. Association between neutropenic complication and other variable.

\begin{tabular}{|c|c|c|c|c|c|}
\hline & & Mean & S.D. & test & $\mathbf{p}$ \\
\hline \multirow[t]{2}{*}{ AGE } & Neutropenic complication & 49.92 & 8.758 & 0.191 & 0.663 \\
\hline & No Neutropenic complication & 49.24 & 8.198 & & \\
\hline \multirow[t]{2}{*}{ tumor size } & Neutropenic complication & 2.23 & 0.688 & 0.176 & 0.676 \\
\hline & No Neutropenic complication & 2.28 & 0.615 & & \\
\hline \multirow{2}{*}{ Lymph nodes } & Neutropenic complication & 13.37 & 5.586 & 4.752 & $0.031^{*}$ \\
\hline & No Neutropenic complication & 11.38 & 4.084 & & \\
\hline \multirow[t]{2}{*}{ Pretreatment ANC } & No delay & 4393.66 & 1781.880 & 11.516 & 0.001 \\
\hline & Delayed & 3409.10 & 1350.584 & & \\
\hline \multirow[t]{2}{*}{ Pretreatment HB } & Neutropenic complication & 11.963 & 1.3927 & 1.325 & 0.252 \\
\hline & No Neutropenic complication & 11.660 & 1.4862 & & \\
\hline \multirow{2}{*}{ Pretreatment Platelet } & Neutropenic complication & 337.15 & 104.742 & 3.795 & 0.054 \\
\hline & No Neutropenic complication & 303.02 & 85.436 & & \\
\hline \multirow{2}{*}{ first cycle nadir ANC } & Neutropenic complication & 1997.45 & 1383.213 & 24.231 & $0.0001^{*}$ \\
\hline & No Neutropenic complication & 1026.21 & 606.288 & & \\
\hline \multirow{2}{*}{ first cycle HB nadir } & Neutropenic complication & 11.277 & 1.0424 & 0.950 & 0.332 \\
\hline & No Neutropenic complication & 11.081 & 1.1640 & & \\
\hline \multirow{2}{*}{ first cycle plat nadir } & Neutropenic complication & 278.71 & 89.915 & 4.285 & $0.041^{*}$ \\
\hline & No Neutropenic complication & 240.31 & 112.668 & & \\
\hline
\end{tabular}

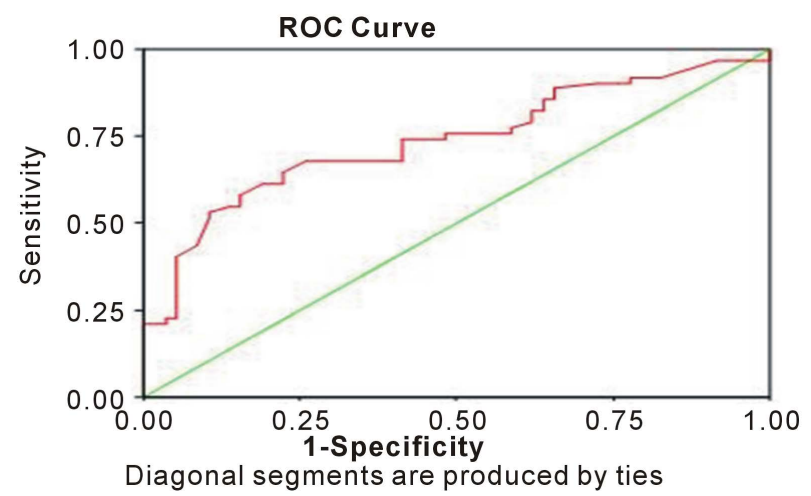

Figure 2. ROC curve of first cycle ANC nadir.

Table 4. Menopausal status in relation to neutropenic complications.

\begin{tabular}{ccccc}
\hline \multirow{2}{*}{ MENOPOSE } & \multicolumn{3}{c}{ dose delayed or FN } & \multirow{2}{*}{ Total } \\
\cline { 2 - 4 } & & No delay & Delayed & \\
\hline Premenopausal & No. & 31 & 35 & 66 \\
& $\%$ & $51.7 \%$ & 58.3 & 55 \\
Postmenopausal & No. & 29 & 25 & 54 \\
& $\%$ & $48.3 \%$ & 41.7 & 45 \\
Total & No. & 60 & 60 & 120 \\
& $\%$ & $100.0 \%$ & $100.0 \%$ & $100.0 \%$ \\
$\mathrm{X}^{2}$ & \multicolumn{4}{c}{0.901} \\
$\mathrm{p}$
\end{tabular}

tropenia and its consequences to develop predictive models capable of identifying patients at greater risk for such complications.But unfortunately most of these stu-
Table 5. Multivariate logistic regression model predicting neutropenic complications.

\begin{tabular}{|c|c|c|c|c|c|}
\hline & \multicolumn{3}{|c|}{$\begin{array}{l}\text { Unstandardized Standardized } \\
\text { Coefficients Coefficients }\end{array}$} & \multirow[t]{2}{*}{ 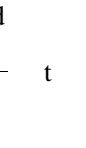 } & \multirow{2}{*}{ Sig. } \\
\hline & B & $\begin{array}{l}\text { Std. } \\
\text { Error }\end{array}$ & Beta & & \\
\hline (Constant) & 0.957 & 0.175 & & 5.470 & 0.000 \\
\hline Lymph nodes $(\mathrm{N})$ & -0.014 & 0.010 & -0.136 & -1.410 & 00.161 \\
\hline $\begin{array}{c}\text { Pretreatment first cycle } \\
\text { ANC }\end{array}$ & 0.000 & 0.000 & -0.140 & -1.461 & 10.147 \\
\hline $\begin{array}{c}\text { Pretreatment first cycle } \\
\text { platelets }\end{array}$ & 0.001 & 0.001 & 0.142 & 1.196 & 0.234 \\
\hline first cycle platelets nadir & -0.001 & 0.001 & -0.109 & -1.012 & 20.314 \\
\hline first cycle ANC nadir & 0.000 & 0.000 & -0.345 & -3.445 & 50.001 \\
\hline
\end{tabular}

dies were retrospective and conclusive results were limited because of the different study designs, small patients number and diverse chemotherapy regimens used in these studies.

Silber et al. were first published a retrospective model for predicting the risk of neutropenia from first cycle nadir in 1998. These authors reviewed the cases of breast cancer patients who received adjuvant chemotherapy with either FAC (fluorouracil, doxorubicin, and cyclophosphamide) or standard CMF regimens. The study demonstrated statistically significant association between first cycle ANC nadir and occurrence of neutropenic complications ( $p=0.0001)$. It also demonstrated statistically significant association between first cycle hemoglobin nadir and occurrence of neutropenic complications $(p=0.0001)$. In compare to our study the treatment re- 
gimen different among population group (56 patients received CMF and 39 patients received FAC regimen). Thirty nine patients received chemotherapy concurrent with radiotherapy which is not the stander and these patients received from 7 to 8 cycles of chemotherapy instead of standard 6 cycles. The dose reduction would be taken if the prior cycle ANC nadir approached 500 cells $/ \mu \mathrm{L}$ which is not common practice in oncology community. Dose delayed would be taken if the ANC was less than 2000 cells $/ \mu \mathrm{L}$ on the first day of the cycle which is quiet high value and most of guideline used ANC less than 1500 cells $/ \mu \mathrm{L}$ as cut off point for dose delay.

Rivera et al. conducted prospective trial at MD Anderson cancer center to validate the predictive role of the first cycle nadir in neutrophil count and occurrence of various neutropenic complications that was described by Silber et al. The study was conducted on 143 patients. All patients were treated with the FAC. They demonstrated statistically significant association between first cycle ANC nadir and occurrence of neutropenic complications $(p=0.0001)$. In compare to our study; chemotherapy dose reduction if the previous cycle ANC nadir was less than 500 cells $/ \mu \mathrm{L}$ which is not common practice in oncology community. Moreover the definition of febrile neutropenia was 1000 cells $/ \mu \mathrm{L}$ with a temperature of more than $38.3^{\circ}$ which is quite high from most of guidelines which use ANC cut off point less than 500 cells $/ \mu \mathrm{L}$ as definition of febrile neutropenia.Also they did not describe the if there were any association between other factors and occurrence of neutropenic complications.

In this study patient age was not a statistically significant association with occurrence of neutropenic complications $(p=0.663)$ although other retrospective study shows statistically significant association between patients' age and neutropenic complications.In one retrospective series that include 1243 breast cancer patients received adjuvant chemotherapy, patient age $\geq 65$ years old had statistically significantassociation with decrease the relative dose intensity of delivered chemotherapy $(\mathrm{p}=0.001)$.

Pretreatment hemoglobin level was not a statistically significantassociation with occurrence of neutropenic complications $(p=0.252)$ in this study, although other retrospective studies demonstrated association between pretreatment hemoglobin level and neutropenic complications. In one retrospective study that included 577 patients with intermediate-grade, non-Hodgkin's lymphomas (NHL) who were received treatment with CHOP chemotherapy. The risk of febrile neutropenia was statistically significant association with baseline hemoglobin $<12 \mathrm{~g} / \mathrm{dl}(\mathrm{p}=0.018)$. Another study that carried out on 1617 patients (704 NHL and 913 early-stage breast cancer) who received initial chemotherapy at 16 community and academic oncology practices between 1991 and 1999 demonstrated statistically significantassociation between neutropenic complications and baseline hemoglobin $<12.0$ g/dL (OR 1.90; 95\% CI: 1.41 - 2.58). The lack of statistically significantcorrelation in our study may attribute to small number of neutropenic events.

First cycle hemoglobin nadir was not statistically significantcorrelation with occurrence of neutropenic complication ( $\mathrm{p}=0.332$ ) in this study, however in Silber predictive modelthere wasstatistically significantassociationbetween first cycle hemoglobin nadir and occurrence of neutropenic complications $(\mathrm{p}=0.0001)$.

\section{Conclusion}

In the view of discussion above, the first cycle absolute neutrophil counts nadir provides useful information on the risk of future neutropenia, febrile neutropenia and dose delay or reduction. Other factors, such as pretreatment absolute neutrophil count, number of involved LN, pretreatment platelet count and first cycle platelet nadir can be used to predict occurrence of neutropenic complications in breast cancer patients undergoing chemotherapy. Further studies among different populations with different tumor types and different treatment regimens are needed to confirm the results and explore other factors which may contribute to occurrence of neutropenic complications.

\section{REFERENCES}

[1] L. Jardines, B. G. Haffty, et al., "Breast Cancer Overview,” In: R. Pazdur, L. R. Coia, W. J. Hoskins and L. D., Wagman, Eds., Cancer Management a Multidisciplinary Approach, 9th Edition, CMPMedica, Lawrence, 2010, p. 163.

[2] National Cancer Institute, "Registry of the National Cancer Institute (2002-2003),” NCI, Cairo, 2004, p. 45.

[3] Early Breast Cancer Trialists' Collaborative Group, "Effects of Chemotherapy and Hormonal Therapy for Early Breast Cancer on Recurrenceand 15-Year Survival: An Overview of the Randomized Trials,” Lancet, Vol. 365, No. 9472, 2005, p. 1687. http://dx.doi.org/10.1016/S0140-6736(05)66544-0

[4] A. H. Partridge, H. J. Burstein and E. P. Winer, "Side Effects of Chemotherapy and Combined Chemohormonal Therapy in Women with Early-Stage Breast Cancer," Journal of the National Cancer Institute, Vol. 2001, No. 30, 2001, pp. 135-142. http://dx.doi.org/10.1093/oxfordjournals.jncimonographs. $\underline{\mathrm{a} 003451}$

[5] B. F. Cole, R. D. Gelber, S. Gelber, et al., "Polychemotherapy for Early Breast Cancer: An Overview of the Randomised Clinical Trials with Quality-Adjusted Survival Analysis,” Lancet, Vol. 358, No. 9278, 2001, p. 277. http://dx.doi.org/10.1016/S0140-6736(01)05483-6 
[6] J. Naurois, N. Basso, et al., "Management of Febrile Neutropenia: ESMO Clinical Practice Guidelines," Annals of Oncology, Vol. 21, No. 5, 2010, pp. 252-256. http://dx.doi.org/10.1093/annonc/mdq196

[7] W. T. Hughes, D. Armstrong, G. P. Bodey, et al., "2002 Guidelines for the Use of Antimicrobial Agents in Neutropenic Patients with Cancer," Clinical Infectious Diseases, Vol. 34, No. 6, 2002, p. 730. http://dx.doi.org/10.1086/339215

[8] G. Bonadonna, E. Brusamolino, P. Valagussa, et al., “Combination Chemotherapy as an Adjuvant Treatment in Operable Breast Cancer," The New England Journal of Medicine, Vol. 294, 1976, pp. 405-410. http://dx.doi.org/10.1056/NEJM197602192940801

[9] G. Bonadonna, P. Valagussa, A. Moliterni, et al., “Adjuvant Cyclophosphamide, Methotrexate, and Fluorouracil in Node-Positive Breast Cancer,” The New England Journal of Medicine, Vol. 332, 1995, pp. 901-906. http://dx.doi.org/10.1056/NEJM199504063321401

[10] J. N. Timmer-Bonte, E. M. Adang, H. J. Smit, et al., "Cost-Effectiveness of Adding Granulocyte Colony-Stimulating Factor to Primary Prophylaxis with Antibiotics in Small-Cell Lung Cancer,” Journal of Clinical Oncology, Vol. 24, No. 19, 2006, p. 2991. http://dx.doi.org/10.1200/JCO.2005.04.3281

[11] C. L. Vogel, M. Z. Wojtukiewicz, R. R. Carroll, et al., "First and Subsequent Cycle Use of Pegfilgrastim Prevents Febrile Neutropenia in Patients with Breast Cancer: A Multicenter, Double-Blind, Placebo-Controlled Phase III Study,” Journal of Clinical Oncology, Vol. 23, No. 6, 2005, p. 1178. http://dx.doi.org/10.1200/JCO.2005.04.3281

[12] J. H. Silber, M. Fridman, R. S. DiPaola, et al., "FirstCycle Blood Counts and Subsequent Neutropenia, Dose Reduction, or Delay in Early-Stage Breast Cancer Therapy," Journal of Clinical Oncology, Vol. 16, 1998, pp. 2392-2400.

[13] E. Rivera, M. H. Erder and G. N. Hortobagyi, "FirstCycle Absolute Neutrophil Count Can Be Used to Improve Chemotherapy-Dose Delivery and Reduce The Risk of Febrile Neutropenia in Patients Receiving Adjuvant Therapy: A Validation Study," Breast Cancer Research, Vol. 5, No. 5, 2003, pp. R114-R120. http://dx.doi.org/10.1186/bcr618 